\title{
Fabrication of soft gold microelectrode arrays as probes for scanning electrochemical microscopy
}

\author{
Andreas Lesch ${ }^{\mathrm{a}}$, Dmitry Momotenko ${ }^{\mathrm{b}}$, Fernando Cortés-Salazar ${ }^{\mathrm{b}}$, Ingo Wirth ${ }^{\mathrm{c}}$, Ushula Mengesha Tefashe ${ }^{\mathrm{a}}$, \\ Frank Meiners ${ }^{a}$, Britta Vaske ${ }^{\mathrm{a}}$, Hubert H. Girault ${ }^{\mathrm{b}}$, Gunther Wittstock ${ }^{\mathrm{a}, *}$ \\ ${ }^{a}$ Carl von Ossietzky University of Oldenburg, Faculty of Mathematics and Natural Sciences, Center of Interface Science, Department of Pure and Applied Chemistry, D-26111 \\ Oldenburg, Germany \\ ${ }^{\mathrm{b}}$ Ecole Polytechnique Fédérale de Lausanne, Laboratoire d'Electrochimie Physique et Analytique, Station 6, CH-1015 Lausanne, Switzerland \\ ${ }^{\mathrm{c}}$ Fraunhofer Institute for Manufacturing Technology and Applied Materials Research (IFAM), Wiener Strasse 12, D-28359 Bremen, Germany
}

\section{A R T I C L E I N F O}

\section{Article history:}

Received 31 August 2011

Received in revised form 30 November 2011

Accepted 1 December 2011

Available online 10 December 2011

\section{Keywords:}

Flexible electrodes

Microelectrode array

Scanning electrochemical microscopy

Gold electrodes

Non-contact printing

\begin{abstract}
A B S T R A C T
Soft linear gold microelectrode arrays for high throughput scanning electrochemical microscopy (SECM) imaging were fabricated using the Aerosol Jet ${ }^{\circledR}$ printing technology. Nanoparticulate gold ink was printed on polyimide Kapton $\mathrm{HN}^{\circledR}$ thin films. After sintering, a $200 \mathrm{~nm}$ thick Parylene $\mathrm{C}$ coating was deposited to cover and seal the gold tracks. A cross-section of the array of microelectrodes was exposed by laser cutting using an ArF excimer laser beam directed onto a metallic mask. Cyclic voltammograms, approach curves and SECM images in feedback mode demonstrate the capability of the arrays as SECM probes. Reactivity imaging of a platinum band structure on glass was performed with Parylene $\mathrm{C}$ coating facing the substrate providing an almost constant working distance. The softness of the array leads to a bending and allows scanning in contact mode like brushing the sample surface. For hard surfaces such as array electrode structures and similar materials, this occurs without detectable damage to the sample.
\end{abstract}

(C) 2011 Elsevier B.V. All rights reserved.

\section{Introduction}

Scanning electrochemical microscopy (SECM) is a scanning probe technique that is used for the local surface characterization with respect to electrochemical reactivity [1-5]. Typically, the diffusion-controlled steady-state Faradaic current at an amperometric ultramicroelectrode (UME) is recorded as a function of the position relative to a specimen surface. Thus, if the sample produces or consumes chemical species converted at the UME, the reaction rate at the specimen will affect the current at the UME probe allowing the reactivity characterization of the surface. The feedback mode [6] and the sample generation-tip collection mode [1] are the most popular strategies of coupling the Faradaic reaction at the UME probe with the reactions occurring at the sample. There is a broad range of literature reports that have expanded the applicability of the technique to other interfaces such as liquid/liquid or liquid/gas [7], and have introduced new working modes such as the tip generation-sample collection $[8,9]$ or the redox competition mode [10] for the investigation of specific catalytic reactions. Instrumental developments are actively pursued in different laboratories to increase the lateral resolution by integrating electrodes of nanometer extension into probes for scanning force

\footnotetext{
* Corresponding author. Tel.: +49 (0) 441798 3971; fax: +49 (0) 4417983979.

E-mail address: gunther.wittstock@uni-oldenburg.de (G. Wittstock).
}

microscopy [11-15], using the electrodes also as shear force sensors [16-18] or combining it with scanning ion-conductance microscopy [19]. These combined techniques help to maintain a constant working distance above curved substrates and samples that have a roughness and/or topography that are not negligible compared to the electrode size.

Another direction of development came from the growing insight that pressing societal needs require the discovery of previously unknown materials [20]. This includes catalysts for energy conversion [21,22] and materials for light harvesting [23]. The potential for electrochemical investigation of material libraries has been demonstrated, in which the inspection of specimens coated with the material libraries that measure square millimeters or square centimeters rather than a few square micrometers is required. Similar requirements exist for scanning tissue samples, proving the absence of defects in technical coatings, readout of electropherograms [24], protein arrays [25,26] or human fingerprints [27]. Although SECM provides the basic methodology for extracting critical information from such samples, its application may be very cumbersome for the following reasons. Scanning large samples requires long times since the lateral scan rate is limited due to convective effects at higher lateral translation rates [28]. Longer imaging times may lead to changes in the solution composition by solvent evaporation or (photo)chemical degradation of solutes and specimens, for instance. Secondly, the current in SECM 
images depends on the local reactivity of the sample and the distance between the UME and the specimen. As a consequence, changes on the probe-substrate distance during scanning could cause topographic artifacts that complicate data interpretation. In most cases one would use a sample surface that is smooth compared to the size of the UME and align it as close as possible to the horizontal $(x, y)$ plane of the positioning system. However, real samples are usually not smooth and the substrate leveling process becomes demanding (and time consuming) if the scan length becomes longer. For some applications a shear force system may alleviate this problem [18] but it requires additional instrumentation and further restricts the lateral scan rate due to the comparatively long time constants of the positional feedback operating with typically rather small signal changes.

Recently our laboratories have demonstrated SECM imaging of large sample areas with flexible probes [29]. Initially the concept of fabricating soft UME probes by filling laser-ablated microchannels in a polymer sheet with heat-curable carbon ink, sealing by lamination and exposure of the cross-section by mechanical or laser-assisted cutting had been demonstrated [30]. The probes could be used while the edge of the insulating lamination layer contacts the specimen surface providing an almost constant working distance. We call this the "contact mode". In principle all SECM working modes mentioned above can be combined with the contact mode though only the feedback mode has been used so far. The mechanical contact between the lamination layer of the probe and the specimen surface causes the flexible probe to bend similar to a giant scanning force microscope cantilever. The distance between the working electrode and the substrate is defined by the thickness of the lamination foil, the exact geometry under which the cross-section was exposed and the inclination angle of the SECM holder under which the soft probe is approached. This is conceptually similar to the combined SECM-AFM probes of Kranz et al. [13], and similar probes proposed afterwards $[14,15]$ in which the distance of the active electrode area to the sample is kept approximately constant by a thorn that mechanically contacts the surface permanently or intermittently. The flexible polymeric probes were able to accommodate sample tilts without any additional electronic instrumentation or preliminary sample leveling [30].

Subsequently, a number of improvements and expansions of the concept have been introduced. The mentioned soft stylus probe was multiplexed to a soft linear array of eight individually addressable carbon microelectrodes [31]. A $0.9 \times 0.4 \mathrm{~cm}^{2}$ area was scanned in contact mode in only $5 \mathrm{~h}$ by such an array with an electrode-to-electrode distance of $500 \mu \mathrm{m}$. Multiple tips have been previously used mainly in scanning force microscopy imaging to scan large areas in shorter time without compromising the resolution [32,33]. Arrays of individually addressable microelectrodes for SECM had been reported. Meyer et al. [34] imaged oxygen, hydrogen peroxide and glucose distributions by an array of 400 platinum microelectrodes which was fabricated as a part of a monolithic sensor chip in a semiconductor process. Barker et al. showed the feasibility of a linear array of 16 platinum microelectrodes on an insulating chip for parallel SECM imaging [35]. For our flexible electrodes, the insulating lamination layer was replaced by a much thinner Parylene $C$ coating that provided for smaller working distances and therefore higher current contrast. The control software SECMx was expanded to allow acquisition from up to 32 probe electrodes that are kept at the same working potential and read via a commercial multiplexing unit. For each of the probe electrodes offsets in the horizontal directions ( $x$ and $y$ ) are stored from which the exact location of each measured point can be reconstructed. The slightly different sizes and shapes of the individual probes of an array are adjusted by an offset current and a scale factor in order to produce similar corrected currents from all electrodes of an array. The inclination angle of the probe electrode was optimized and the SECMx software was expanded by the possibility to retract the array so that it is freely suspended in solution during the reverse scan in horizontal direction (lift-off). With this options millimeter sized images of entire human fingerprints were obtained and showed both the macroscopic features (ridge ending, bifurcations) as well as microscopic intrinsic ridge features such as pores size and position [29]. Another development concerns the possibility to deliver a microliter droplet of the electrolyte solution to the specimen surface avoiding the need of mounting samples in a liquid cell [36]. The versatility is further increased by probes that deliver and aspirate the mediator solution via separate microchannels that can also be interfaced to complementing analytical instrumentation [37].

As an alternative to carbon ink filled microchannels the deposition of materials on plastic substrates can be performed by noncontact printing techniques, such as inkjet or aerosol based printing which are less expensive, less time consuming and allow high substrate flexibility. Inkjet printing can be used to fabricate 2D and $3 \mathrm{D}$ structures. The technique is similar to the one used in common inkjet printers and allows the use of special inks containing materials like nanoparticles or biomolecules. For delivering ink droplets thermal, piezoelectric or electrostatic driven systems are mostly used [38]. Inkjet printing and sintering of nanoparticulate gold inks on flexible substrates was reported by Huang et al. using polyethylene terephthalate and polyester substrates for flexible electronics [39], while Jensen et al. printed on polyimide films to fabricate an array of gold electrodes for manufacturing an immunosensor for the electrochemical detection of the cancer biomarker interleukin-6 in serum [40]. A different concept of printing nanoscale suspensions is the Aerosol Jet ${ }^{\circledR}$ printing technology (formly termed maskless mesoscale materials deposition, $M^{3} D$ ) [41]. An aerosol is generated by an ultrasonic transducer or a pneumatic nebulizer depending on the viscosity of the ink. The aerosol that consists of femtoliter droplets is carried by an inert gas stream into the printing module, where a co-axial flow of the aerosol stream and an enclosing sheath gas stream is formed. Due to co-axial focusing a nozzle with a diameter of usually $100 \mu \mathrm{m}$ in the printhead can generate line widths of less than $10 \mu \mathrm{m}$. Printing structures on planar and non-planar substrates is easily possible due to a nozzle substrate distance of up to $5 \mathrm{~mm}$. A shutter is installed to interrupt the continuous aerosol flow. Post-treatment of the printed areas is performed by heating in an oven or (especially for low temperature substrates) by a laser system that is integrated in the printing device which generates a continuous laser beam of the same dimensions as the printed track width. Applications of Aerosol Jet ${ }^{\circledR}$ printing technology have been found for the fabrication of cell arrays and printing of biomolecules or metal inks for sensing purposes [42]. Front side metallization of silicon solar cells with $5 \mathrm{~cm} \times 5 \mathrm{~cm}$ silver grids as a first layer of a 2-layer contact was also reported [43].

In the present work, a new methodology for the fabrication of soft linear microelectrode arrays is introduced. Aerosol printing technology was used to deliver droplets containing gold nanoparticles directly on polyimide thin films. After solvent evaporation and sintering a thin Parylene $C$ coating sealed the nanoparticulate tracks. For characterization and SECM imaging in the contact mode, the active electrode area of the electrode array was exposed by laser ablation or razor blade cutting.

\section{Experimental}

\subsection{Chemicals}

Ferrocene methanol (FcMeOH, $\geqslant 97 \%$, Sigma-Aldrich, Buchs, Switzerland, or Alfa Aesar, Karlsruhe, Germany), ethanol (VWR 
International S.A.S, Fontenay-sous-Bois, France), acetonitrile (VWR International S.A.S, Fontenay-sous-Bois, France), tetrabutylammonium trifluoromethanesulfonate ( $\geqslant 99 \%$, Sigma-Aldrich, Steinheim, Germany), $\mathrm{KNO}_{3} \quad(\geqslant 99 \%$, Sigma-Aldrich, Buchs, Switzerland, or Carl Roth, Karlsruhe, Germany) and gold nanoparticle ink (gold nanoparticle content 15 wt.\%, Nanocolltech Ltd., Hungary) were used as received. Deionized water was produced by a Milli-Q plus 185 model (Millipore, Zug, Switzerland) or by Purelab $^{\circledR}$ Classic (Elga LabWater, United Kingdom). Gold microelectrode arrays were fabricated on $125 \mu \mathrm{m}$ thick polyimide Kapton $\mathrm{HN}^{\circledR}$ films from Goodfellow (Huntingdon, England). Connection pads were fabricated by Electrador carbon ink (Electra Polymer \& Chemicals Ltd., Roughway Mill, Dunk Green, England).

\subsection{Preparation of platinum electrodes}

Pt bands on glass were prepared by standard photolithographic processes and employed as test samples for surface reactivity imaging. The width, pitch and thickness of the Pt bands were $100 \mu \mathrm{m}, 100 \mu \mathrm{m}$ and $100 \mathrm{~nm}$, respectively. Positive and negative feedback were tested over a substrate with insulating (glass) and conductive $(\mathrm{Pt})$ regions that was fabricated in the same manner as the Pt band structure.

\subsection{Soft microelectrode array preparation}

Eight nanoparticle gold ink lines of approximately $6 \mathrm{~cm}$ length and with a midpoint-midpoint distance of $500 \mu \mathrm{m}$ were printed on $125 \mu \mathrm{m}$ thick polyimide films using an Aerosol Jet ${ }^{\circledR}$ printer work station (Optomec Inc., Singer, Albuquerque, USA) similar to a previous report [42]. After printing, the gold ink tracks were sintered in an oven under atmosphere for $2 \mathrm{~h}$ at $400{ }^{\circ} \mathrm{C}$ in order to eliminate ink additives, which are used for stabilization of Au nanoparticles in the ink, and to achieve electrical conductivity. Depending on the printing parameters, the received line widths were either $40 \mu \mathrm{m}$ or $50 \mu \mathrm{m}$ for all tracks in one array. A $200 \mathrm{~nm}$ thick Parylene $C$ coating was deposited over the gold tracks by using a Parylene depositing system (Comelec SA, La Chaux-deFonds, Switzerland). Electronic connection pads for the individual gold tracks were formed by manually applying conductive carbon ink followed by curing at $80^{\circ} \mathrm{C}$ for $1 \mathrm{~h}$. A cross-section of the gold array structure was exposed by UV laser ablation through a metallic mask using a $193 \mathrm{~nm}$ ArF excimer laser beam (Lambda Physik, Göttingen, Germany, fluence $=0.2 \mathrm{~J}$, frequency $=50 \mathrm{~Hz}$ ) as described previously $[30,44]$ or by cutting with a razor blade.

\subsection{SECM measurements}

SECM measurements were carried out using a custom-built SECM setup running under SECMx software [45] comprising an Ivium CompactStat (bi)potentiostat (Ivium Technologies, Eindhoven, The Netherlands), supplemented by an Ivium WE32 multiplexer unit allowing the operation of up to 32 individually addressable working electrodes against one counter (Pt wire, $\mathrm{CE}$ ) and one reference electrode ( $\mathrm{Ag}$ wire as quasi-reference electrode, $\mathrm{Ag}-\mathrm{QRE}$ ). The same potentials were applied and held at the same time to all individual electrodes and for all electrodes current values were measured simultaneously. All potentials are reported with respect to the Ag-QRE. The platinum sensor structure was used as unbiased sample to be imaged and was mounted at the bottom of a flat cell whose tilt with respect to the horizontal scanning plane of the soft linear array was eliminated by a Zaber tilt table (Zaber Technologies Inc., Vancouver, Canada). The probe itself was mounted in a custom-built SECM holder in such a way that the Parylene C coating was facing the sample surface. Thanks to an inclination angle of $70^{\circ}$ in the probe holder, the degree and bending direction of the probe was preset before scanning in contact mode (Supporting information SI-1). Approach curves were recorded until all individual electrodes mechanically touched the sample surface, which is clearly detectable by a sudden leveling of the individual current values. After a line scan in contact regime, the electrode array was retracted from the sample surface until it was freely suspended in solution and the reverse horizontal scan was performed [31]. The acquired data from the forward line scans was used to plot the 3D image by using the custom-made software MIRA [46].

\subsection{SECM shear force experiments}

Shear force-based SECM images were performed with a SECMx system consisting of a DS4L controller for shear force distance control with SXM software, version 0.19f (Anfatec Instruments AG, Oelsnitz, Germany). The DS4L control software runs on an independent personnel computer (PC). The vertical position control signal was fed into the piezo controller and amplifier E-662 (Physik Instrumente, Karlsruhe, Germany) operated in open loop. This controller drove a linear piezo stage with a translation range of $80 \mu \mathrm{m}$ and an internal position sensor (type P-780.20, Physik Instrumente, Karlsruhe, Germany). The basic SECM system consisted of a positioning system (Märzhäuser GmbH \& Co KG, Wetzlar, Germany) and a custom-made analog bipotentiostat (M. Schramm, University of Düsseldorf, Germany) interfaced to the PC controlling the SECM via an analog-digital/digital-analog board (PCI-DAS 1602/16, PlugIn Electronic GmbH, Eichenau, Germany). The signal from the position sensor of the linear piezo stage was also read by the analogdigital board and used to construct a topographical image.

A $700 \mathrm{~nm}$ diameter platinum nanoelectrode (with a quartz glass shield of $18 \mu \mathrm{m}$ ) was fabricated following the general recommendations by Etienne et al. [47]. Specifically, a laser puller (P-2000, Sutter Instrument Co., Novato, CA) was used to seal the Pt wire and glass capillary repeating five times a sequence of $15 \mathrm{~s}$ heating and 20 s cooling (laser puller program parameters were heat, 640; Filament, 4; velocity, 100; delay, 100; pull, 1) while pulling was prevented by a mechanical barrier. After obtaining a well sealed capillary, the barrier was removed and the capillary was pulled apart (heat, 640; Filament, 2; velocity, 100; delay, 100; pull, 220). The tip was then processed according to Ref. [47]. The excitation of mechanical vibration of such electrodes was made with a sine wave ( $395 \mathrm{kHz}$ frequency and $579 \mathrm{mV}$ amplitude) provided by the DS4L controller and directed to a piezo plate. The vibration was detected by a similar plate glued to the SECM tip as recommended by Ballesteros-Katemann et al. [18]. The three-electrode cell was completed by an Ag-QRE and a Pt wire as CE. The sample was unbiased.

\subsection{AFM and CLSM measurements}

Atomic force microscopy (AFM) in the Tapping Mode ${ }^{\mathrm{TM}}$ of the sintered gold tracks on polyimide films was done in air at room temperature in order to obtain the topographic information of the sintered tracks using a Nanoscope IIIA controller, a Dimension 3100 sample stage, a Dimension SPM head scanner and a RTESP tip (all Veeco Instruments Inc., Plainview, NY, USA). A $256 \times 256$ pixel image of a $100 \mu \mathrm{m} \times 100 \mu \mathrm{m}$ area was recorded with a scan rate of $0.1 \mathrm{~Hz}$. Image flattening was performed using the Nanoscope software (V5.30r3sr3) with a first order polynomial that eliminates the $z$ offset between line scans, and the tilt in each line scan, by calculating a first-order, least-squares fit for the selected segment and then subtracting it from the line scan. A confocal laser scanning microscope (CLSM, TCS SP2 AOBS, Leica Microsystems GmbH, Wetzlar, Germany) was used in reflection mode with an Ar laser $(488 \mathrm{~nm})$. 


\subsection{Investigation of the force by the soft probe on the sample}

The force of the soft probe brought onto the sample was estimated by two experimental approaches: Measuring the mass of the array in contact with the sample and measuring the resonance frequency of a vibrating array.

\subsection{Numerical simulations}

Finite element method (FEM) simulations were performed with the software COMSOL Multiphysics (version 3.5a) for solving of Fick's laws of diffusion. It was run on a Linux Ubuntu 8.04 platform with four-core Mac Pro 2.66 GHz CPU and 9.8 GB of RAM.

\section{Results and discussion}

\subsection{Probe characterization}

A top view of one Aerosol Jet ${ }^{\circledR}$-printed and sintered array of eight gold tracks is shown in Fig. 1a. The array was cut out from the foil along the dashed line to mount it in a custom-made SECM holder. The adhesion of the gold tracks is high enough to sustain scratching with a surgical scalpel (result not shown). The midpoint-midpoint distance between two adjacent electrode tracks is $500 \mu \mathrm{m}$ and the width of an individual line in this array is approximately $50 \mu \mathrm{m}$ (Fig. 1b), i.e. the inter electrode spacing is $450 \mu \mathrm{m}$. This distance was selected in order to minimize the overlap of the diffusion layers between adjacent individual electrodes. It has been shown before that diffusional cross-talk in microelectrodes arrays is important. Barker et al. used a distance of $120 \mu \mathrm{m}$ for $10 \mu \mathrm{m}$ diameter electrode to have a smaller diffusion layer thickness than half of the distance between two neighboring electrodes [35]. Our previous experiments and simulations on flexible carbon electrode arrays showed that diffusional overlapping among neighboring electrodes (i.e. $500 \mu \mathrm{m}$ midpoint-midpoint distance and $40 \mu \mathrm{m}$ electrode width) produced a current difference of $2.1 \%$ in comparison with a single electrode, but showed no difference among inner and outer electrodes [31].
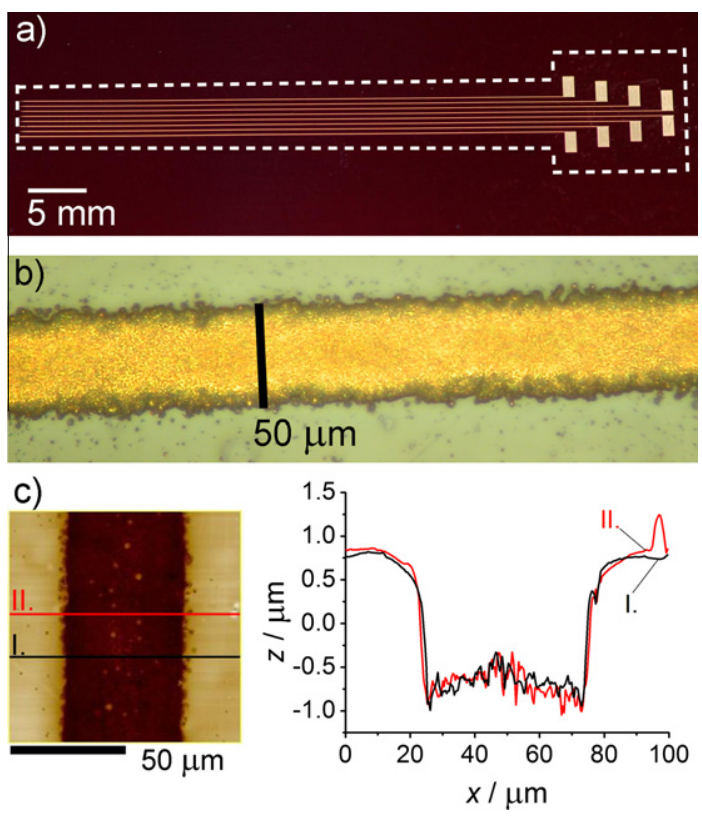

Fig. 1. (a) Optical photograph of printed gold structure on polyimide film after sintering. The dashed line indicates the shape of the array after fabrication. (b) Optical micrograph of a single sintered gold track. (c) Topographic AFM image of a sintered gold track and two exemplary topographic line scans.
The height of the tracks is difficult to investigate because the polyimide film undergoes relaxation during sintering at $400^{\circ} \mathrm{C}$. The film thickness increases except for the areas covered by gold [48]. As a consequence the gold tracks appear to be slightly recessed from the surrounding polymer sheet. Fig. 1c shows a $100 \mu \mathrm{m} \times 100 \mu \mathrm{m}$ topographic AFM image of a sintered gold track and two horizontal line scans along the black and red line in the image. It can be seen clearly that the polyimide swelled next to the gold track and that the gold track is nearly $1.3 \mu \mathrm{m}$ recessed in the polymer film. The sintered nanoparticles are readily identifiable. The thickness of the gold tracks can be obtained from images of the cross-sections after Parylene C coating (vide infra). Additionally in the red colored line scan a spike can be obtained on pure Kapton $\mathrm{HN}^{\circledR}$. As discussed later these features are observed all over the polymer sheet and are considered as real.

Each gold line is terminated by a larger rectangular gold area (Fig. 1a, right side). These areas were enlarged by carbon ink to enable contacting in an electrode holder used previously for electrode arrays with carbon tracks [31]. This holder provides for individual contact of each electrode to a multiplexed potentiostat.

The electrochemical reactivity of the sintered gold tracks without Parylene $C$ coating was investigated using shear force SECM in the feedback mode. A nanoelectrode was scanned in a constant distance over the sample comprising two recessed gold tracks. The topography and the electrochemical reactivity were recorded simultaneously by storing the position signal of the piezo actuator used for maintaining a constant working distance (i.e. the topography signal) and the nanoelectrode current (electrochemical reactivity). Fig. 2 shows the topographic signal and the SECM feedback current from a $700 \mu \mathrm{m}$ line scan (a) and a stacked 3D plot of the topography and SECM feedback image of a $700 \mu \mathrm{m} \times$ $500 \mu \mathrm{m}$ scanned area (b). The measured currents over gold are significantly larger than over polyimide. A homogeneous contribution of the electrochemical reactivity of the sintered nanoparticles can be seen. This proves the electrical contact to all of the nanoparticles and a homogeneous removal of the organic additives of the used gold ink. In comparison to the AFM image in Fig. 1c the roughness of the sintered gold nanoparticles cannot be detected by the used shear force tip in the topographic image. The spikes on pure Kapton $\mathrm{HN}^{\circledR}$ are observed in the forward and in the corresponding reverse scan image and also in an AFM image on pure polyimide (SI-2). They are therefore considered as real features rather than noise or artifacts from the electronic feedback used in the shear force system.

The cross-section of the layered structure with Parylene $C$ coating was exposed by laser cutting or razor blade cutting. The cross section of the printed gold tracks forms the active electrode areas and have the shape of a band electrode with about $1.67 \mu \mathrm{m}$ width and $40 \mu \mathrm{m}$ length for the array shown in Fig. 3a. Six adjacent microelectrodes of the array were electronically connected and each one was electrochemically characterized by cyclic voltammetry (CV) in the bulk of an aqueous $2 \mathrm{mM}$ FcMeOH solution at $10 \mathrm{mV} \mathrm{s}^{-1}$ (Fig. 3b). A sigmoidal response indicates steady state behavior. The low hysteresis is indicative of an acceptable sealing between the gold tracks, Kapton $\mathrm{HN}^{\circledR}$ films, and the covering Parylene $\mathrm{C}$ coating. Capacitive currents are higher than expected from the geometric area but this is not surprising given the preparation from a nanoparticle aerosol. The different steady-state currents of the individual electrodes reflect the sligho0tly various electrode areas and geometries. This is a result of the printing and sintering processes as well as of the cutting procedure. In addition, two CVs are vertically shifted to higher or lower current values. These current offsets can arise from limitations using a multiplexer. In order to investigate the quality of the active electrode areas, a wave-slope analysis of the CVs was performed. Plotting the applied tip potential $E_{\mathrm{T}}$ of all electrodes $k$ as a function of $\log \left[\left(i_{\mathrm{T}, \infty, k}-i_{\mathrm{T}, k}\right) / i_{\mathrm{T}, k}\right](\mathrm{SI}-3)$ where $i_{\mathrm{T}, \infty, k}$ 


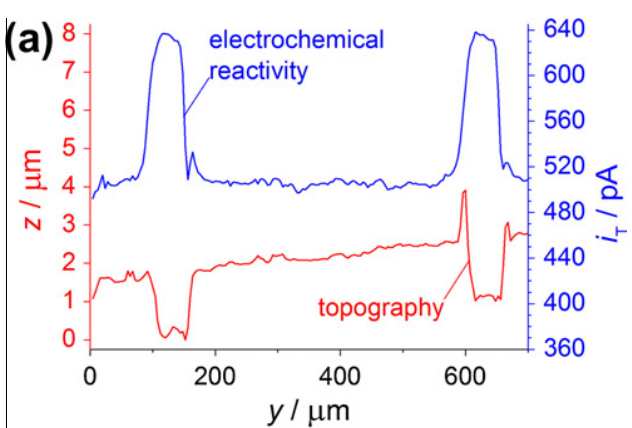

(b)
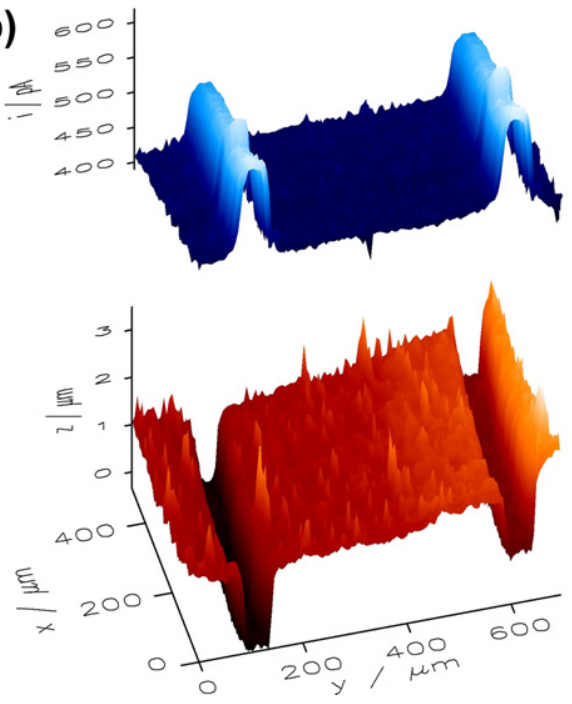

Fig. 2. Shear-force based $\mathrm{SECM}$ in aqueous $2 \mathrm{mM} \mathrm{FCMeOH}$ and $0.1 \mathrm{M} \mathrm{KNO}_{3}$; $E_{\mathrm{T}}=0.3 \mathrm{~V}$, translation rate $25 \mu \mathrm{m} \mathrm{s}^{-1}$, step size $5 \mu \mathrm{m}$. Line scan (a) and SECM image (b) over two sintered gold tracks with simultaneously recorded topographic and surface reactivity information.

is the diffusion-limited current of electrode $k$ in bulk solution at steady state conditions and $i_{\mathrm{T}, k}$ is the current at a defined given potential $E_{\mathrm{T}}$ of electrode $k$ in bulk solution. The calculated slopes of the electrodes $(-68.6 \mathrm{mV} ;-61.5 \mathrm{mV} ;-60.5 \mathrm{mV} ;-60.8 \mathrm{mV}$; $-60.8 \mathrm{mV} ;-62.3 \mathrm{mV}$ ) have slightly higher absolute values than the theoretical value of $-59.1 \mathrm{mV}$ [49] for a reversible system. This is most likely due to the internal resistance of the printed gold structure and limitations of the electron transfer rate at the exposed gold structure. The resistance of the gold structures was measured using a four point probe. The contact pins with $2 \mathrm{~mm}$ separation gave a resistance $R=8.13 \Omega$. The specific resistance $\rho$ is calculated by Eq. (1) assuming a homogeneous current distribution inside the gold track.

$\rho=R \cdot \frac{A}{l}$

Here $l=2 \mathrm{~mm}$ is the length and $A=83.63 \mu \mathrm{m}^{2}$ is the area of the cross-section of the measured track yielding a specific resistance of $3.4 \times 10^{-7} \Omega \mathrm{m}$. Therefore, the measured conductivity is $2.94 \times 10^{6} \mathrm{~S}^{-1} \mathrm{~m}^{-1}$ which is $6.5 \%$ of the value for bulk gold material.

FEM simulations of one gold microelectrode were performed assuming a gold track of $40 \mu \mathrm{m}$ width and $1.67 \mu \mathrm{m}$ height (SI-4). The simulated steady-state current of $4.3 \mathrm{nA}$ in an aqueous $2 \mathrm{mM}$ FcMeOH solution corresponds very well to the experimentally observed steady state currents in the CVs taking into account the various electrode geometries.

The fabricated gold electrodes can also be used in organic solvents such as acetonitrile as shown in Fig. 3c. For this experiment, (a)

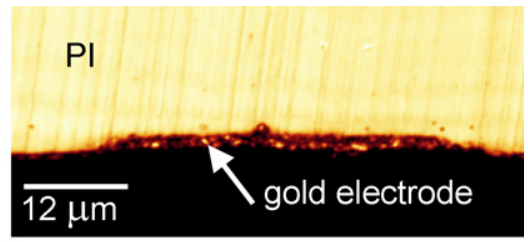

(b)

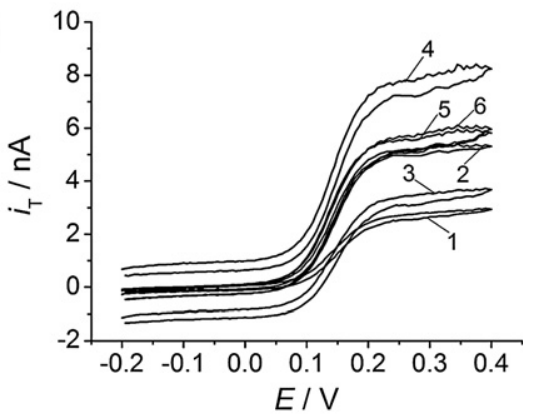

(c)

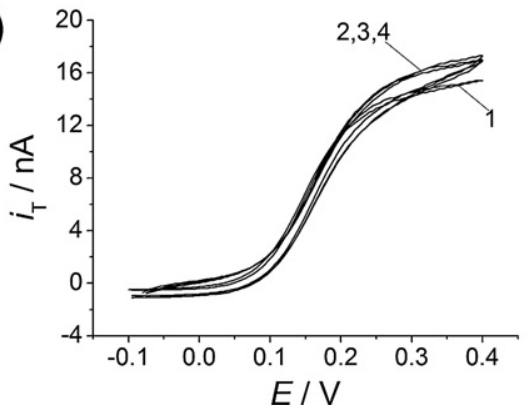

Fig. 3. (a) CLSM reflection image of one cross-section of one active electrode within a printed gold electrode array covered with Parylene $\mathrm{C}$ coating. Cutting was performed with a razor blade. (b) Cyclic voltammograms of six gold microelectrodes of one linear array after laser cutting in aqueous $2 \mathrm{mM} \mathrm{FcMeOH}$ and $0.1 \mathrm{M}$ $\mathrm{KNO}_{3}$. Potential scan rate was $10 \mathrm{mV} \mathrm{s}^{-1}$. (c) Cyclic voltammograms of one gold microelectrode after blade cutting in $2 \mathrm{mM} \mathrm{FcMeOH}$ and $0.1 \mathrm{M}$ tetrabutylammonium trifluoromethanesulfonate dissolved in acetonitrile. Each of the cycles after $0 \mathrm{~min}$ (curve 1), $30 \mathrm{~min}$ (2), $60 \mathrm{~min}$ (3) and $90 \mathrm{~min}$ (4) was performed directly after renewal of the solution. Potential scan rate was $20 \mathrm{mV} \mathrm{s}^{-1}$.

several CV's were taken with only one gold microeletrode in a $2 \mathrm{mM}$ FcMeOH solution with $20 \mathrm{mV} \mathrm{s}^{-1}$. The first scan shows a nice steady state behavior (curve 1 in Fig. 3c). Within 10 min the response changes a bit (curve 2 in Fig. 3c) before it stays constant over the whole investigated time period of $2 \mathrm{~h}$ (curves 3-4 in Fig. 3c). Each of the CVs in Fig. 3c was made directly after renewal of the electrolyte solution in the cell in order to provide comparable current values without influences by evaporation of the organic solvent. In the following all experiments were performed in aqueous solutions.

\subsection{Approach curve characterization and current calibration}

SECM approach curves were performed in aqueous FcMeOH mediator solution either over an insulating (glass, Fig. 4a) or a conducting (platinum, Fig. 4b) surface while the microelectrode array was biased at $E_{\mathrm{T}}=0.3 \mathrm{~V}$ to cause diffusion-controlled oxidation of $\mathrm{FcMeOH}$. The vertical position of the array at the mechanical contact point with the sample surface was defined as $h_{\mathrm{P}}=0 \mu \mathrm{m}$. Therefore, $h_{\mathrm{P}}$ becomes negative after touching the surface. Due to slightly different sizes, geometries and Parylene $C$ coating thicknesses the current values vary as already shown in the CVs. In Fig. 4a typical negative feedback behavior indicated by a decreasing current value is observed when the array approached the insu- 

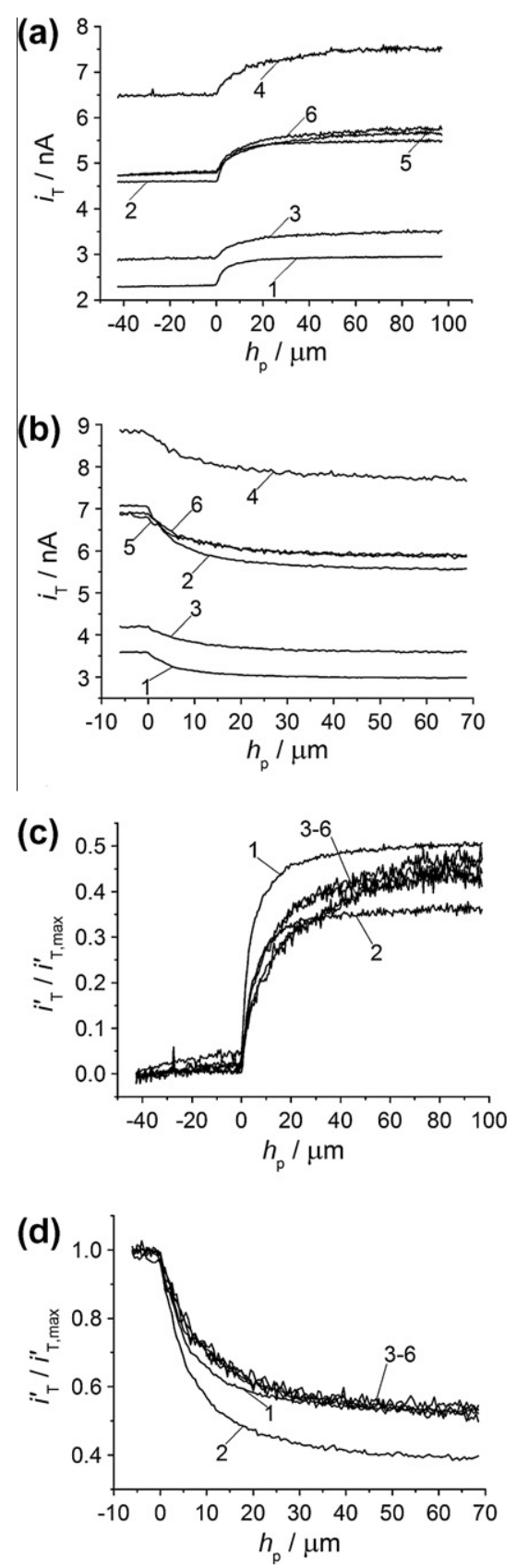

Fig. 4. Experimental approach curves of an array with six electrically connected gold electrodes over insulating glass (a) and unbiased platinum (b) in aqueous $2 \mathrm{mM} \mathrm{FcMeOH}$ and $0.1 \mathrm{M} \mathrm{KNO}_{3} ; E_{\mathrm{T}}=0.35 \mathrm{~V}$ after laser cutting, translation rate $2 \mu \mathrm{m} \mathrm{s}^{-1}$. (c) and (d) show the currents after application of the correction factors that account for the different sensitivities and offsets of the individual electrodes (Eq. (5)).

lating surface. After contact with the sample the array bends and starts to slide. Due to an almost constant working distance, the current values do not change significantly during this sliding. Similarly, when approaching to a conductive substrate the recorded currents for all the gold microelectrodes increase as the recycling of FcMeOH is achieved until the array physically touches the surface (Fig. 4b).

In order to construct SECM images from experiments with array probes, the individual electrodes must have the same response characteristics. This is very difficult to achieve with the employed preparation protocols. In order to work with non-perfect arrays we proposed a correction formula that is applied to all currents of each electrode of an array to obtain similar responses. Finding the correction values was done initially by interactively adjusting the factors after recording an image until a uniform response was achieved above structures that extend through the sample regions imaged by different electrodes. This gave convincing results but was nevertheless not satisfactory because it introduced an arbitrary element and could be time-consuming. Therefore, a new approach was sought that depends only on experiments that are readily performable or are anyway part of a typical SECM routine. The contact point can be easily identified from the approach curves in Fig. 4a. For $h_{\mathrm{p}} \leqslant 0 \mu \mathrm{m}$ the currents of each electrode remain constant within the noise level. The current differences between electrodes at $h_{\mathrm{P}} \leqslant 0$ are mainly due to the current offset observed in Fig. $3 b$ and small variations of the electrode-sample geometry caused for instance by slight variations of the Parylene $C$ coating thickness or the electrode-sample alignment. The latter current differences can be overcome if a current leveling procedure is performed by assuming that the experimental response above glass (negative feedback) and Pt (positive feedback) represents the minimum or maximum current possible for a given individual working electrode in the contact regime. Therefore, the first step is to calibrate each individual sensor $k$ to get a current $i_{\mathrm{T}, k}^{\prime}$ by a current offset $i_{\mathrm{T}, \mathrm{offs}, k}$ to the measured current $i_{\mathrm{T}, k}$ (Eq. (2)), where $i_{\mathrm{T}, \mathrm{offs}, k}$ is the negative of the experimentally observed current $i_{\mathrm{T}, \mathrm{nf}, k}$ for negative feedback after mechanically touching the insulating substrate $\left(h_{\mathrm{P}} \leqslant 0 \mu \mathrm{m}\right)$ (Eq. (3)).

$i_{\mathrm{T}, k}^{\prime}=i_{\mathrm{T}, k}-i_{\mathrm{T}, \mathrm{offs}, k}$

$i_{\mathrm{T}, \mathrm{offs}, k}=i_{\mathrm{T}, \mathrm{nf}, k}\left(h_{\mathrm{P}} \leqslant 0 \mu \mathrm{m}\right)$

This procedure sets the current over insulating regions to $0 \mathrm{nA}$. Afterwards, a scale factor $s_{k}$ for each electrode is obtained as the reciprocal of the maximum offset-corrected current of the approach curve performed over the conducting substrate for the $k$-th sensor (Eq. (4)).

$s_{k}=\frac{1}{i_{\mathrm{T}, \max , k}^{\prime}}=\frac{1}{i_{\mathrm{T}, \max , k}-i_{\mathrm{T}, \mathrm{off} s, k}}$

This procedure sets $i_{\mathrm{T} k}^{\prime} / i_{\mathrm{T} \max k}^{\prime}$ to 1 for diffusion-controlled positive feedback and to 0 for negative feedback in contact regime. All measured currents were transformed by Eq. (5) using the current offsets and scale factors listed in Table 1.

$\frac{i_{\mathrm{T}, k}^{\prime}}{i_{\mathrm{T}, \max , k}^{\prime}}=\left(i_{\mathrm{T}, k}^{\prime}-i_{\mathrm{T}, \mathrm{offs}, k}^{\prime}\right) \cdot s_{k}$

This routine was applied not only to the currents measured in the approach curves, but also to the subsequently recorded SECM images (vide infra). The transformed approach curves are shown in Fig. 4c and d, where almost equal current values are observed when the array is in mechanical contact with either glass or platinum. However, when the array is in the solution bulk the lowest

Table 1

Current offsets, scale factors and positional offsets of the individual sensors determined by approach curve analysis.

\begin{tabular}{llllc}
\hline Electrode $k$ & $i_{\mathrm{T}, \mathrm{off}, k}(\mathrm{nA})^{\mathrm{a}}$ & $s_{k}\left(\mathrm{nA}^{-1}\right)^{\mathrm{a}}$ & $x_{\text {off }, k}(\mu \mathrm{m})$ & $y_{\text {offs }, k}(\mu \mathrm{m})$ \\
\hline 1 & $2.29(1.90)$ & 0.77 & 0 & 0 \\
2 & $4.59(4.10)$ & $0.40(0.41)$ & 20 & 500 \\
3 & 2.90 & 0.76 & 30 & 1000 \\
4 & 6.48 & 0.42 & 40 & 1500 \\
5 & 4.74 & 0.47 & 50 & 2000 \\
6 & $4.72(4.25)$ & $0.46(0.47)$ & 55 & 2500
\end{tabular}

${ }^{\mathrm{a}}$ values in brackets represent manually adjusted values used for Fig. $5 \mathrm{c}$ (see text for details). 
calibrated currents $i_{\mathrm{T}, k}^{\prime} / i_{\mathrm{T}, \mathrm{max}, k}^{\prime}$ differ up to $29 \%$ from the highest one among the individual electrodes of one array. This could be a consequence of several parameters such as misalignment between the sample plane and the array or by slightly different geometries of the active electrode areas. Moreover, as the soft gold array is intended to be used in a contact regime, the calibration procedure outlined above is adequate to level the current values of all the sensors in the array. Such a calibration approach is applicable if samples contain areas where such a calibration can be done on specific regions, e.g. glass and Pt regions that are touched by each array element.

In previous papers [31] we have experimented with different ways of dealing with the combined effect of distance variation between individual electrodes, current offsets (caused by instrumental imperfections) and sensitivities (caused by size variation of the exposed electrode area). In previous reports we estimated those values based on receiving a consistent imaging on model samples. We were not satisfied with this approach as it introduced an arbitrary element and could be time consuming until self-consistent estimates were found. The values for $i_{\mathrm{T}, \text { off }}$ and $i_{\mathrm{T}, \infty}$ are not separately accessible. Here we propose a more operational approach in which the contrast between inert insulating sample regions and conducting sample regions is normalized, circumventing separate estimates of $i_{\mathrm{T}, \infty}$ and the current offset in the solution bulk as done earlier. Please note that the $i_{\mathrm{T}, \mathrm{offs}, k}$ as defined in Eq. (3) is not identical to a current offset in the solution bulk used in [31] as it also compensates for variation in the working distance between individual electrodes. We want to stress clearly, that soft microelectrode array probes are not suitable for quantitative kinetic studies in which knowledge of exact working distances, electrode shapes and bulk currents is indispensible.

\subsection{High throughput SECM imaging}

In order to demonstrate the capabilities of the Aerosol Jet ${ }^{\circledR}$ printed SECM probe array, SECM imaging of the electrochemical reactivity of a platinum band structure supported on glass was performed with the gold soft array in contact mode. The test sample was chosen because it contains clearly differentiated conducting and insulating regions in the micrometer range for surface reactivity characterization by feedback mode. The flexible probe was pressed against the substrate surface until a defined negative $h_{\mathrm{p}}=-42.5 \mu \mathrm{m}$ was reached. A constant working distance was provided by the stiffness of the polyimide film, the Parylene $C$ coating thickness and the defined angle $\left(70^{\circ}\right)$ of the array in the holder (SI1 ). Afterwards, a line scan in $x$ direction was performed in such a way that the gold soft probe was sliding and every $10 \mu \mathrm{m}$ the movement is interrupted and the currents of all microelectrodes are recorded while the probe is resting. At the end of the line scan the soft probe was retracted $300 \mu \mathrm{m}$ before moving back in "contactless mode" $\left(h_{\mathrm{P}}>0\right)$ to the starting position of the line scan. The stroke height value was chosen in order to avoid any mechanical damage of the soft probe by crashing the sample surface during the reverse scan and allowing higher translation rates for the reverse scans. Before starting the next line scan in contact mode, the array probe was displaced by a step of $10 \mu \mathrm{m}$ in the $y$ direction and was moved down until $h_{\mathrm{p}}=-42.5 \mu \mathrm{m}$. By repeating this procedure 50 times, a sample area of $3 \mathrm{~mm} \times 3 \mathrm{~mm}$ was scanned and a reactivity image with 90,300 experimental data points is constructed (Fig. 5). The SECM image shown in Fig. 5a was build up by combining the six images recorded by each microelectrode and taking into account the appropriate $y$ position given by the interelectrode distances (offsets $y_{\text {off,k }}$ ). The imaging time was $4 \mathrm{~h}$ and $50 \mathrm{~min}$, which is much less than the $20 \mathrm{~h}$ and $30 \mathrm{~min}$ needed to scan the same area with only one microelectrode using the same parameters except the lift-off mode. The exact imaging time de-
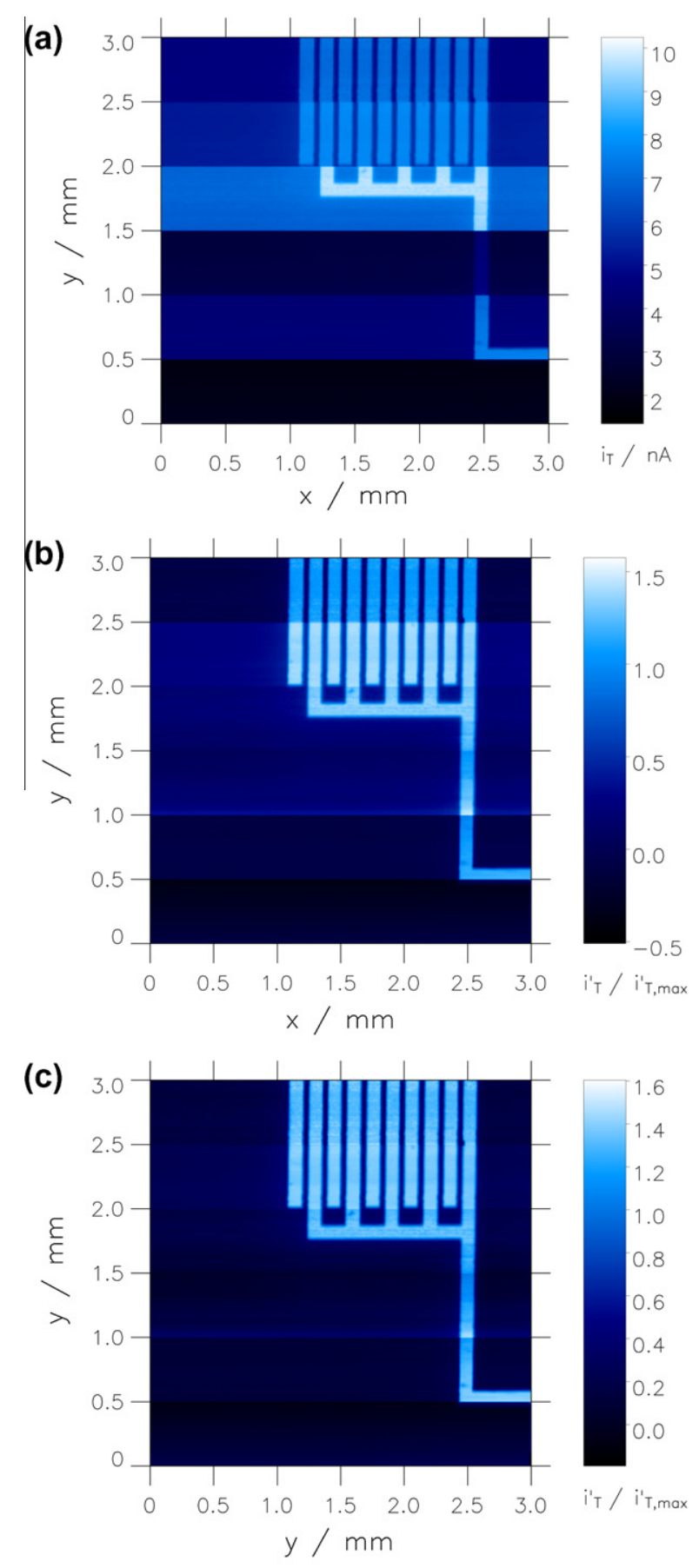

Fig. 5. (a) SECM feedback image in aqueous $2 \mathrm{mM} \mathrm{FCMeOH}$ and $0.1 \mathrm{M} \mathrm{KNO}_{3}$ of an unbiased platinum structure on insulating glass substrate $(3 \mathrm{~mm} \times 3 \mathrm{~mm})$ with an array of six gold microelectrodes (midpoint-midpoint distance $=500 \mu \mathrm{m}$ ) after laser cutting. $h_{\mathrm{p}}=-42.5 \mu \mathrm{m}$, step size $=10 \mu \mathrm{m}$, and translation rate $v_{\mathrm{T}}$ of the recorded forward scan in contact mode was $25 \mu \mathrm{m} \mathrm{s}^{-1}$, while in contactless mode was $250 \mu \mathrm{m} \mathrm{s}^{-1}$ with a retract and re-approach speed of $250 \mu \mathrm{m} \mathrm{s}^{-1}$ and $10 \mu \mathrm{m} \mathrm{s}^{-1}$, respectively. (b) SECM feedback image from transformed currents considering the slightly different electrochemically responses of the individual microelectrodes and the values from Table 1 (details in 3.3). (c) SECM feedback image from transformed currents using minor manual adjustments to $i_{\text {off, } k}$ and $s_{k}$ values (in brackets) for some electrodes in Table 1 (details in 3.3).

pends on the height by which the probe is retracted during the reverse line scans and on the time for reading the current values at each position with the digital Ivium multiplexer (approx. $100 \mathrm{~ms}$ for six sensors in comparison to $20 \mathrm{~ms}$ for a single electrode). Therefore, the imaging time is reduced by a factor of 4.2 when using an array of six microelectrodes compared to a single micro- 
electrode. In Fig. 5a, the regions of high current values represent the Pt band structures, which are able to recycle the redox mediator. In contrast, low current values are recorded when the array probe is scanned over the glass region due to hindered redox mediator diffusion. Additionally, as the soft gold array is scanned in contact mode with the Parylene $C$ coating facing the sample, a very small working distance is achieved that remains almost constant during the scanning process as the soft probe can follow the sample topology. For this reason, no topographic artifacts are observed in any of the frames shown in Fig. 5a, even if the frames do not have the same current values. In order to calibrate Fig. 5a, Eq. (5) was applied to all the current values. The result of this calibration is shown in Fig. 5b, where similar current values are obtained above glass and Pt among the different microelectrodes. Slight current variation and in particular corrected currents larger than unity are observed most likely because solvent evaporation increased mediator concentration during the course of the experiment. Additionally, the offset values used for current calibration were taken from approach curve experiments that might differ slightly from forward line scans in the bending degree and sliding direction of the probe (SI-1). Nevertheless, Fig. 5b shows the capabilities of the soft gold array and the above mentioned calibration routine for imaging localized surface reactivity. An SECM image with almost equal responses for all microelectrodes can be obtained by a minor manual correction similar to our previous procedure (Fig. 5c) [31]. The modified current offsets and scale factors are given in brackets in Table 1 . Additionally, the $x$ positions of an individual sensor $k$ was corrected by applying a positional offset $x_{\text {offs }, k}$. These minimal variations in the lateral positions along the direction of a line scan are caused by a deviation from a $90^{\circ}$ angle between the direction of the gold tracks and the direction of laser cutting that is difficult to prevent or by deviation in mounting the probe array holder. The corrected image with homogenously distributed currents corroborates the potential of the demonstrated gold electrode array for SECM imaging in contact regime of the electrochemical reactivity of a metal deposited on an insulator (Fig. 5c).

The main advantage that soft arrays provided is the possibility to scan larger areas in less time avoiding problems such as electrode fouling, sample aging or drastical solvent evaporation. This advantage can be extended to even larger sample areas than the one given by the microelectrode array width, if the multiple imaging routine integrated in SECMx is employed allowing the automatically patching of different images [31]. In the present case, a $6 \mathrm{~mm} \times 6 \mathrm{~mm}$ image is acquired with 50 line scans of $6 \mathrm{~mm}$ length and a space increment of $10 \mu \mathrm{m}$ between line scans. By using the multiple imaging routine a large displacement of $3 \mathrm{~mm}$ along the $y$ axis can be performed after recording an area of $3 \mathrm{~mm} \times 6 \mathrm{~mm}$ followed by scanning a second adjacent image frame of $3 \mathrm{~mm} \times 6 \mathrm{~mm}$ (without double scanning of sample regions). This routine can be repeated as many times as desired opening the possibility of imaging very large areas. In the present work, a four times larger area of the sample shown in Fig. 5 was scanned consecutively without sample cleaning, array cutting or renewing of the solution. The obtained image contained 360,600 data points and took $18 \mathrm{~h}$ and $30 \mathrm{~min}$ to record (Fig. 6a). Correction of currents in Fig. 6a was done by using Eq. (5), but does not provide convincing results because the response of the individual electrodes changed over time. For instance, the currents from sensor number 4 increases progressively during the experiment (see consecutive line scans of sensor 4 in Fig. 6b), while for sensor number 3 the current decreases (see consecutive line scans of sensor 3 in Fig. 6c). The current increase with time can be explained by the increase of the redox mediator concentration as a consequence of solvent evaporation during the experiment over the prolonged imaging time. The current decrease could be a result of the deactivation (a)

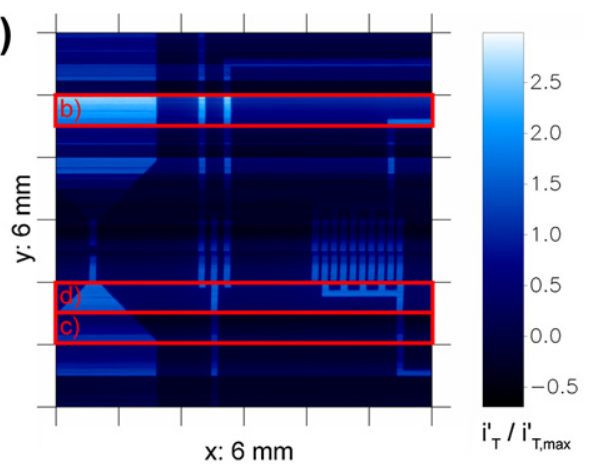

(b)

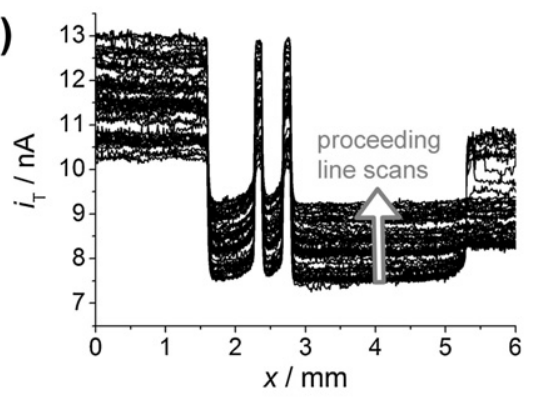

(c)

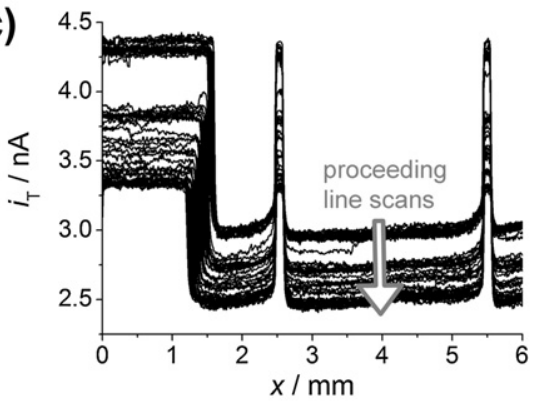

(d)

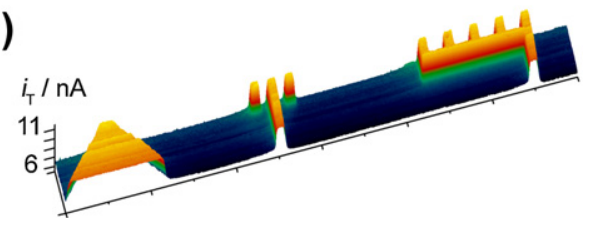

Fig. 6. (a) Transformed multiple SECM feedback image of an area of $6 \mathrm{~mm} \times 6 \mathrm{~mm}$ of the platinum band structure on glass with the array of six microelectrodes and a midpoint-midpoint distance of $500 \mu \mathrm{m}$ after laser cutting performed in an aqueous $2 \mathrm{mM} \mathrm{FcMeOH}$ and $0.1 \mathrm{M} \mathrm{KNO}_{3}$ solution. $h_{\mathrm{p}}=-42.5 \mu \mathrm{m}$, step size $=10 \mu \mathrm{m}$, and translation rate $v_{\mathrm{T}}=25 \mu \mathrm{m} \mathrm{s}^{-1}$. The response of some microelectrodes changed during the image. Either continuously increasing (b, sensor 4, uncorrected currents) or decreasing currents (c, sensor 3, uncorrected currents) were obtained. (d) Image of 50 lines recorded by sensor 4 before the large step (uncorrected currents). Tick length of $x$ and $y$ axis is $500 \mu \mathrm{m}$.

of individual electrodes by adsorption of impurities present in the media or precipitation over the electrode of the highly concentrated supporting electrolyte or redox mediator. The gold inks contain organic components that may not be removed completely by sintering and may contaminate the working solution as well. The Parylene $C$ coating is subjected to abrasion when sliding over the sample for prolonged time. Abraded parts of the Parylene $C$ coating may mechanical obstruct the small working area of the probe.

Despite these unfavorable circumstances, the surface reactivity is clearly resolved and the electrochemical response of all channels is clearly detectable even after nearly $24 \mathrm{~h}$ of continuous imaging without renewal of the solution or of the active electrode area. 
To improve the quality of the large step images a renewal of the active electrode areas can be done by laser or blade cutting before each experiment or by simply electrochemical cleaning between experiments or during reverse scans. It is also possible to use only one channel of the array for imaging or to use the data of individual electrodes for plotting. Fig. 6d shows the first 50 line scans of sensor 4 in a surface plot. The image shows a high contrast image of a still large sample area of $0.5 \mathrm{~mm} \times 6 \mathrm{~mm}$ with a total of 30,050 data points. The edge definition is high.

The investigated sample made of thin deposited platinum lines on glass is much harder than the used probe itself and, therefore, not sensitive to scratches during scanning in contact mode. By contrast the probe, especially the Parylene $C$ coating that is touching the sample surface during scanning is abraded during scanning (SI-5, Figs. SI-5-2b and SI-5-2c). This does not affect the insulation of the gold tracks as they are recessed by $1.3 \mu \mathrm{m}$ from the surrounding material as can be seen from the AFM image (Fig. 1c) and the scanning electron microscopic image of the cross section (SI-5, Figs. SI-5-2a and SI-5-2d). In order to calculate the approximate pressure exerted on the sample by the soft probe the vertical force and the contact area were estimated by two experimental approaches. They are based on direct force measurement with a balance and determination of the resonance frequency (SI-5). The spring constant from direct force measurement was $k=2.97 \mu \mathrm{N} \mu \mathrm{m}^{-1}$ corresponding to a force at vertical displacement $h_{\mathrm{p}}=-42.5 \mu \mathrm{m}$ used in SECM imaging of $F=-k \times h_{\mathrm{p}}=126.2 \mu \mathrm{N}$. The measured resonance frequency of $f=18.75 \mathrm{~Hz}$ and the mass $m_{\mathrm{vib}}=75 \mathrm{mg}$ of the vibrating part of the array yielded a spring constant of $k=4 \pi^{2} \times f^{2} \times m_{\text {vib }}=1.04 \mathrm{~kg} \mathrm{~s}^{-2}$ and a force of $F=-k \times h_{\mathrm{p}}=44.2 \mu \mathrm{N}$. Both experiments show results of the same magnitude and demonstrate that the force on the sample is relatively weak. This becomes clear when considering the contact area $A_{\text {cont }}$ and comparing the pressure $p=F / A_{\text {cont }}$ to conventional AFM experiments. $A_{\text {cont }}$ is difficult to determine because the laser assisted cutting leads to an accumulation of ablated material at the rim of the cut area (SI-5-2). However, SEM images before and after scanning (SI-5-2) show that some probe polymeric material was removed or deformed during scanning and allows assuming a contact area at the end of the performed experiment of $A_{\text {cont }}=1840 \mu \mathrm{m}^{2}$. This yields vertical pressure of $p=68.6 \times 10^{3} \mathrm{~N} \mathrm{~m}^{-2}$ and $24.0 \times 10^{3} \mathrm{~N} \mathrm{~m}^{-2}$ for the direct force measurement and the resonance experiment, respectively. Conventional AFM tips in contact mode apply forces in the range of $1 \mathrm{nN}<F<100 \mathrm{nN}$ on a contact area with a diameter of $2-10 \mathrm{~nm}$ [50]. Taking the smaller limit of the force $(1 \mathrm{nN})$ and the upper limit of the contact area $\left(78 \mathrm{~nm}^{2}\right)$ yields a $p=1.3 \times 10^{7} \mathrm{~N} \mathrm{~m}^{-2}$ which is four orders of magnitude higher than the pressure exerted by the soft array. However, for sensitive samples like protruding biological cells the lateral pressure will be of much more significance.

\section{Conclusions}

The present report illustrates the feasibility of using the Aerosol Jet ${ }^{\circledR}$ printing technology for the fabrication of soft gold microelectrode arrays as probes for SECM. Nanoparticle gold ink can be Aerosol Jet ${ }^{\circledR}$ printed on polymeric thin films like polyimide. After sintering, coating with a thin Parylene $C$ film and exposure of a cross-section, gold microelectrode arrays are obtained. High throughput SECM imaging in contact mode of a large sample area with resolved details in the micrometer scale has been achieved with the soft gold arrays. Similar to other flexible probes, the linear gold microelectrode array can be scanned in contact mode while maintaining an almost constant working distance making possible the investigation of tilted or bended surfaces. Introduction of gold as electrode material for flexible electrodes offers the possibility to use a more diverse range of redox mediators and compounds that are difficult to convert at carbon electrodes $\left(\mathrm{O}_{2}, \mathrm{H}_{2} \mathrm{O}, \mathrm{H}_{2} / \mathrm{H}^{+}\right)$or to use organic solvents. The aerosol printing technology provides not only a simpler and more reproducible preparation of soft probes for SECM, but also enables the preparation of probes with different electrode materials. Expansion to further electrode materials can be foreseen. Additionally, the printing technology is suitable for batch production.

\section{Acknowledgements}

The work was jointly supported by Deutsche Forschungsgemeinschaft (Wi 1617/10) and the Fonds National Suisse pour la Recherche Scientifique (Grant No. 20PA21_121570/1) under the title "High throughput SECM imaging". U. M. T. thanks for a grant of German Academic Exchange Service. The technical assistance by Valérie Devaud, Cyrille Hibert (both EPFL), Robert Pomraenke, Renate Kort and Erhard Rhiel (all three University of Oldenburg) is acknowledged.

\section{Appendix A. Supplementary material}

Supplementary data associated with this article can be found, in the online version, at doi:10.1016/j.jelechem.2011.12.005.

\section{References}

[1] R.C. Engstrom, M. Weber, D.J. Wunder, R. Burgess, S. Winquist, Anal. Chem. 58 (1986) 844-848.

[2] A.J. Bard, F.-R.F. Fan, J. Kwak, O. Lev, Anal. Chem. 61 (1989) 132-138.

[3] A.J. Bard, M.V. Mirkin, Scanning Electrochemical Microscopy, Marcel Dekker Inc., New York, Basel, 2001.

[4] G. Wittstock, M. Burchardt, S.E. Pust, Y. Shen, C. Zhao, Angew. Chem. Int. Ed. 46 (2007) 1584-1617.

[5] P. Sun, F.O. Laforge, M.V. Mirkin, Phys. Chem. Chem. Phys. 9 (2007) 802-823.

[6] J. Kwak, A.J. Bard, Anal. Chem. 61 (1989) 1221-1227.

[7] A.L. Barker, M. Gonsalves, J.V. Macpherson, C.J. Slevin, P.R. Unwin, Anal. Chim. Acta 385 (1999) 223-240.

[8] C. Lee, J. Kwak, F.C. Anson, Anal. Chem. 63 (1991) 1501-1504.

[9] J.L. Fernandez, A.J. Bard, Anal. Chem. 75 (2003) 2967-2974.

[10] K. Eckhard, X. Chen, F. Turcu, W. Schuhmann, Phys. Chem. Chem. Phys. 8 (2006) 5359-5365.

[11] J.V. Macpherson, P.R. Unwin, Anal. Chem. 72 (2000) 276-285.

[12] H. Shin, P.J. Hesketh, B. Mizaikoff, C. Kranz, Sens. Actuat. B 134 (2008) 488495.

[13] C. Kranz, G. Friedbacher, B. Mizaikoff, A. Lugstein, J. Smolier, E. Bertagnolli, Anal. Chem. 73 (2001) 2491-2500.

[14] A. Davoodi, J. Pan, C. Leygraf, S. Norgren, Electrochim. Acta 52 (2007) 76977705.

[15] S.E. Pust, M. Salomo, E. Oesterschulze, G. Wittstock, Nanotechnology 21 (2010). 105709-105701-105709-105712.

[16] M. Ludwig, C. Kranz, W. Schuhmann, H.E. Gaub, Rev. Sci. Instrum. 66 (1995) 2857-2860.

[17] P. James, L.F. Garfias-Mesias, P.J. Moyer, W.H. Smyrl, J. Electrochem. Soc. 145 (1998) 64-66.

[18] B. Ballesteros Katemann, A. Schulte, W. Schuhmann, Chem. Eur. J. 9 (2003) 2025-2033.

[19] Y. Takahashi, A.I. Shevchuk, P. Novak, Y. Murakami, H. Shiku, Y.E. Korchev, T. Matsue, J. Am. Chem. Soc. 132 (2010) 10118-10126.

[20] A.J. Bard, J. Am. Chem. Soc. 132 (2010) 7559-7567.

[21] J.L. Fernandez, D.A. Walsh, A.J. Bard, J. Am. Chem. Soc. 127 (2005) 357-365

[22] A. Maljusch, T.C. Nagaiah, S. Schwamborn, M. Bron, W. Schuhmann, Anal Chem. 82 (2010) 1890-1896.

[23] J.S. Jang, J. Lee, H. Ye, F.-R.F. Fan, A.J. Bard, J. Phys. Chem. C 113 (2009) 67196724.

[24] M. Zhang, G. Wittstock, Y. Shao, H.H. Girault, Anal. Chem. 79 (2007) 4833 4839.

[25] G. Wittstock, Fresenius J. Anal. Chem. 370 (2001) 303-315.

[26] C. Nunes Kirchner, M. Träuble, G. Wittstock, Anal. Chem. 82 (2010) 2626-2635

[27] M. Zhang, H.H. Girault, Analyst 134 (2009) 25-30.

[28] C. Combellas, M. Fermigier, A. Fuchs, F. Kanoufi, Anal. Chem. 77 (2005) 7966 7975.

[29] F. Cortes-Salazar, D. Momotenko, H.H. Girault, A. Lesch, G. Wittstock, Anal Chem. 83 (2011) 1493-1499.

[30] F. Cortes-Salazar, M. Träuble, F. Li, J.-M. Busnel, A.-L. Gassner, M. Hojeij, G. Wittstock, H.H. Girault, Anal. Chem. 81 (2009) 6889-6896.

[31] F. Cortes-Salazar, D. Momotenko, A. Lesch, G. Wittstock, H.H. Girault, Anal. Chem. 82 (2010) 10037-10044. 
32] S. Hong, C.A. Mirkin, Science 288 (2000) 1808-1811.

[33] P. Vettiger, M. Despont, U. Drechsler, U. Durig, W. Haberle, M.I. Lutwyche, H.E. Rothuizen, R. Stutz, R. Widmer, G.K. Binnig, IBM J. Res. Dev. 44 (2000) 323 340.

[34] H. Meyer, H. Drewer, B. Gründig, K. Cammann, R. Kakerow, Y. Manioli, W. Mokwa, M. Rospert, Anal. Chem. 67 (1995) 1164-1170.

[35] A.L. Barker, P.R. Unwin, J.W. Gardner, H. Rieley, Electrochem. Commun. 6 (2004) 91-97.

[36] F. Cortes-Salazar, A. Lesch, D. Momotenko, J.-M. Busnel, G. Wittstock, H.H. Girault, Anal. Methods 2 (2010) 817-823.

[37] D. Momotenko, F. Cortes-Salazar, A. Lesch, G. Wittstock, H.H. Girault, Anal. Chem. 83 (2011) 5275-5282.

[38] S. Magdassi, Inkjet Printing Technologies, World Scientific Publishing Co., Pte. Ltd., New Jersey, 2010.

[39] D. Huang, F. Liao, S. Molesa, D. Redinger, V. Subramanian, J. Electrochem. Soc 150 (2003) G412-G417.

[40] G.C. Jensen, C.E. Krause, G.A. Sotzing, J.F. Rusling, Phys. Chem. Chem. Phys. 13 (2011) 4888-4894.
[41] G.J. Marquez, M.J. Renn, W.D. Miller, Mater. Res. Soc. Symp. Proc. 698 (2002) 343-349.

[42] I. Grunwald, E. Groth, I. Wirth, J. Schumacher, M. Maiwald, V. Zoellmer, M. Busse, Biofabrication 2 (2010). 014106/014101-014106/014111.

[43] A. Mette, P.L. Richter, M. Hoerteis, S.W. Glunz, Progress Photovoltaics 15 (2007) 621-627.

[44] M.A. Roberts, J.S. Rossier, P. Bercier, H.H. Girault, Anal. Chem. 69 (1997) 2035 2042.

[45] C. Nunes Kirchner, K.H. Hallmeier, R. Szargan, T. Raschke, C. Radehaus, G. Wittstock, Electroanalysis 19 (2007) 1023-1031.

[46] G. Wittstock, T. Asmus, T. Wilhelm, Fresenius J. Anal. Chem. 367 (2000) 346351.

[47] M. Etienne, E.C. Anderson, S.R. Evans, W. Schuhmann, I. Fritsch, Anal. Chem. 78 (2006) 7317-7324.

[48] M.L. Williams, R.F. Landel, J.D. Ferry, J. Am. Chem. Soc. 77 (1955) 3701-3707.

[49] C.G. Zoski, Electroanalysis 14 (2002) 1041-1051.

[50] E. Meyer, H.J. Hug, R. Bennewitz, Scanning Probe Microscopy: The Lab on a Tip, Springer, Berlin [u.a.], 2004. p. 64. 